\title{
Isquemia e reperfusão hepática total em condições de normalidade e sob estado de choque hemorrágico controlado: efeitos no sequestro de neutrófilos no rim do rato ${ }^{1}$
}

\author{
Total hepatic ischemia and reperfusion under normal conditions \\ and submitted to controlled hemorrhagic shock state: \\ effects of neutrophil sequestration in kidney of rats
}

\begin{abstract}
Elcio Shiyoiti Hirano ${ }^{2}$, Mario Mantovani ${ }^{3}$, Rosana Celestina Morandin ${ }^{4}$
1. Pesquisa desenvolvida no Laboratório de Investigação em Cirurgia do Trauma (LICIT) da Disciplina de Cirurgia do Trauma do Departamento de Cirurgia da Faculdade de Ciências Médicas (FCM) da Universidade Estadual de Campinas (Unicamp), no Núcleo de Medicina e Cirurgia Experimental (NMCE). Resumo de Tese de Mestrado defendida e aprovada no Curso de Pós-Graduação em Cirurgia da FCM-Unicamp. 2. Mestre em Cirurgia pela Disciplina de Cirurgia do Trauma da FCM-Unicamp; Médico Assistente da Disciplina de Cirurgia do Trauma da FCM-Unicamp; Médico Assistente e Supervisor da Unidade de Emergência Referenciada do Hospital de Clínicas-Unicamp; Médico Cirurgião e Coordenador do Pronto Socorro do Hospital Municipal de Jaguariúna-SP (Asamas).

3. Professor Titular e Chefe da Disciplina de Cirurgia do Trauma e Chefe do LICIT da FCM-Unicamp.

4. Doutora em Ciências Médicas, área de Anatomia Patológica e bióloga do LICIT.
\end{abstract}

\begin{abstract}
RESUMO
Objetivo: Avaliar os efeitos da isquemia e reperfusão hepática total em condições de normalidade e estado de choque hemorrágico controlado no acúmulo de neutrófilos no interstício do rim do rato. Métodos: Foram utilizados 32 ratos adultos da raça Wistar, machos, divididos em quatro grupos: Grupo Controle (GC), condições de normalidade, submetidos às cateterizações e laparotomia, seguido de um período de 60 minutos de observação; Grupo Choque (GCh): estado de choque hemorrágico controlado (CHC) por 20 minutos (20') em pressão arterial média (PAM) de 40 mmHg, seguido de reanimação volêmica e de 60 minutos de reperfusão (RP60); Grupo Pringle (GP): Manobra de Pringle (MP) por 15 minutos (15’), seguido de RP60; Grupo Choque-Pringle (GCP): estado de CHC por 20" em PAM de $40 \mathrm{mmHg}$, seguido de laparotomia e MP por 15', seguido de RP60. Para caracterização da má perfusão tecidual foram medidos o lactato sangüíneo (LS) e a reserva de base (RB), no início do período de reperfusão (RP0), aos trinta minutos de reperfusão (RP30) e no RP60. Resultados: No RP0 o valor médio do LS e RB apenas o GC apresentou níveis dentro das normalidades. Nos RP30 e RP60 o valores médios do LS e RB do GC demonstrou-se estatisticamente diferente dos demais grupos ( $\mathrm{p}<0,0001)$. Os valores médios de neutrófilos encontrado foram: GC =0,24 ( $\pm 0,28)$; $\mathrm{GCh}=1,06( \pm 0,61)$; GP $=0,18( \pm 0,16)$; GCP $=0,24( \pm 0,19)$. O GC apresentou diferença estatística quando comparado aos demais, os quais não diferiam entre si. Conclusão: O presente modelo experimental demonstrou que o estado de CHC por 20' seguido de RP60 foram os principais responsáveis pelo maior seqüestro de neutrófilo no córtex renal.
\end{abstract}

Descritores: Choque hemorrágico. Neutrófilos. Manobra de Pringle. Rim. Fígado. Experimentação animal.

\begin{abstract}
Purpose: The evaluate the effects of total hepatic ischemia and reperfusion under normal conditions and submitted to controlled hemorrhagic shock state on the accumulation of neutrophils in the cortex renal of rats. Methods: In this experiment 32 male adult Wistar mice, were divided into four groups: Control Group (CG), submitted to the catheterizations and laparotomy, followed by a period 60 minutes of observation; Shock Group (SG): controlled of hemorrhagic shock (CHS) for 20 minutes (20'), mean arterial pressure (MAP) $40 \mathrm{mmHg}$, followed by reanimation and reperfusion for 60 minutes (RP60); Pringle Group (PG): Pringle's Maneuver (PM) for 15 minutes (15’), followed for RP60'; Shock-Pringle Group (SPG): CHS (MAP = $40 \mathrm{mmHg}$ ) for 20' followed by laparotomy and PM for 15', followed for RP60'. To characterize the poor tissular perfusion blood lactate and the base reserve were measured. Results: The means valous neutrophils were: $\mathrm{CG}=0,24( \pm 0,28)$; $\mathrm{SG}=1,06( \pm 0,61) ; \mathrm{PG}=0,18( \pm 0,16)$; $\mathrm{SPG}=0,24( \pm 0,19)$. The CG demonstrated significant neutrophils accumulation in the renal córtex, when compared between others groups. Conclusion: In this experimental study showed that CHS for 20' followed for RP60 was principal factor by major sequestration of neutrophiles in the renal cortex of rats. Key words: Hemorrhagic shock. Neutrophil. Pringle’s maneuver. Kidney. Liver. Animal experimentation.
\end{abstract}




\section{Introdução}

O estado de choque define-se como má perfusão tecidual, cuja etiologia pode ser de origem vasogênica, neurogênica, cardiogênica e hipovolêmica ${ }^{1}$, sendo que no trauma, a causa mais comum é a hipovolemia. No Brasil, as causas externas de mortalidade, que incluem agressões e acidentes de transporte, ocupam o primeiro lugar. Este fato coloca, epidemiologicamente, o trauma como a principal causa de óbito na faixa da terceira década de vida² .

$\mathrm{O}$ choque hemorrágico $(\mathrm{CH})$ produz alterações no sistema cardiorrespiratório com o objetivo de manter a função fisiológica dos órgãos. A principal alteração é o desvio do fluxo sangüíneo da pele, músculos e leito esplâncnico para o coração, pulmão e cérebro ${ }^{3,4}$. Ocorrem outras alterações, como: metabolismo anaeróbico, acidose metabólica, produção de mediadores inflamatórios, lesão celular e a síndrome da disfunção de múltiplos órgãos $(\mathrm{SDMO})^{1}$. Devido a essa redistribuição do fluxo sangüíneo ocorre a isquemia tecidual de outros órgãos, como por exemplo, nos rins ${ }^{5}$. No traumatismo abdominal, o fígado é um dos órgãos mais acometidos ${ }^{6,7}$. No traumatismo hepático complexo, o objetivo inicial é o controle do sangramento para evitar a exsangüinação durante o procedimento cirúrgico. Este controle pode ser realizado através da manobra de Pringle (MP), que consiste na oclusão temporária da tríade portal (artéria hepática, ducto biliar comum e veia porta) localizada no ligamento hepatoduodenal ${ }^{8}$.

A MP interrompe o fluxo sangüíneo produzindo isquemia hepática, bem como congestão venosa no leito esplâncnico, o que resulta posteriormente em isquemia intestinal ${ }^{9,10}$. Com déficit de oxigênio, as células entram em metabolismo anaeróbico, que é o passo inicial para a acidose metabólica, ocorrendo a utilização do piruvato como fonte energética e, conseqüentemente, eleva-se o lactato sérico $(L S)^{11,12}$. O lactato é um ótimo parâmetro bioquímico para se avaliar o estado de choque e a reanimação ${ }^{13-16}$

Na literatura, a reserva de base (RB) também foi utilizada também como parâmetro, na avaliação do choque e reanimação ${ }^{16,17}$. A hipoperfusão renal promove lesão celular, primeiramente no córtex, levando à disfunção do órgão. Essas alterações ocorrem durante a isquemia e também durante o período de reperfusão ${ }^{1,18,19}$. Os eventos que ocorrem na fase de reperfusão caracterizam a denominada síndrome de reperfusão (SR). Na literatura, os estudos sobre a SDMO demonstram a importância da participação do neutrófilo no desenvolvimento dessa síndrome. As funções dos neutrófilos só serão exercidas desde que eles estejam “ativados” por um estímulo. Diante disso, tais células podem se encontrar em quatro situações: quiescentes, sensibilizados, ativados e não responsivos ${ }^{20}$.

Os neutrófilos seqüestram-se de modo indiscriminado em tecidos normal ou lesado e, a partir desse evento, são ativados para produzirem $\mathrm{O}_{2}^{-}$e, conseqüentemente, desenvolver a Síndrome da Resposta Inflamatória Sistêmica (SRIS) seguido da Síndrome de Disfunção de Múltiplos Órgãos (SDMO) ${ }^{21}$. Portanto, além da lesão ocasionada pela isquemia tecidual local ou sistêmica, a ação dos neutrófilos seqüestrados nos tecidos promove uma agressão contínua aos órgãos, representando a alavanca inicial da patogênese da SDMO.

\section{Métodos}

Este trabalho experimental foi realizado no Laboratório de Investigação em Cirurgia do Trauma (LICIT), da Disciplina de Cirurgia do Trauma do Departamento de Cirurgia da Faculdade de Ciências Médicas da Universidade Estadual de Campinas. O protocolo foi conduzido de acordo com os Princípios Éticos na Experimentação Animal adotados pelo Colégio Brasileiro de Experimentação Animal (Cobea), tendo sido aprovado pela Comissão de Ética na Experimentação Animal (CEEA)-IB-Unicamp (protocolo $n^{\circ}$ 144-1). Os animais, fornecidos pelo Centro de Bioterismo da Unicamp - (Cemib), foram mantidos em condições ambientais controladas de luminosidade e temperatura, recebendo dieta padrão do LICIT constituída de ração Nuvilab CR-1 (Nuvital Nutrientes Ltda., Paraná, Brasil) e água ““ad libitum””, por um período de uma semana, para aclimatação, no biotério, antes do início do experimento.

Foram utilizados neste trabalho experimental 32 ratos machos adultos da linhagem Wistar, com pesos que variaram de 185 a 240 gramas, com idade de aproximadamente sete semanas. A seleção dos animais para cada um dos quatro grupos do experimento obedeceu ao método de randomização, que foi utilizado para assegurar que cada animal tivesse uma probabilidade igual ao ser colocado em cada um dos grupos de estudos.

Neste trabalho, foi utilizado o método de alocação sistemática, pois isso garantiu que os grupos fossem do mesmo tamanho. Com base na literatura utilizou-se pela anestesia intraperitoneal com soluções de Cloridrato de (S+) Cetamina, $80 \mathrm{mg} / \mathrm{Kg}$ com Cloridrato de Xilazina 2\%, 10mg/ Kg e atropina, 0,05mg/Kg ${ }^{16,22}$.

Após obter-se o plano anestésico foi realizada a tricotomia no animal , sendo colocado em posição supina sobre uma prancha de aquecimento externo elétrico, realizando o controle da temperatura retal entre $37-38,5^{\circ} \mathrm{C}$.

Foram dissecados e cateterizados os seguintes vasos do lado direito: artérias carótida e femoral e veia jugular. Por intermédio da artéria carótida direita foram realizadas retiradas de sangue em intervalos de dois minutos para o estabelecimento do estado de choque hemorrágico controlado (CHC). Pela veia jugular direita foram realizadas reposições volêmicas de solução de Ringer lactato e sangue total. A pressão arterial média (PAM) foi monitorizada ininterruptamente através da artéria femoral direita. Foram estudados os efeitos após 15' da MP, em estado de hipovolemia ou normovolemia, seguida de RP60. Os animais foram randomizados e divididos em quatro grupos:

Grupo Controle: em condições de normalidade, submetidos às cateterizações e laparotomia, seguido de um período de 60 minutos de observação, correspondente ao período de reperfusão (PR) dos demais grupos.

Grupo Choque: em estado de CHC por sangria, com PAM mantida em 40 mmHg por 20', seguido de reposição volêmica com solução de Ringer Lactato (RL) aquecido (39$40^{\circ} \mathrm{C}$ ) e sangue total até atingir a PAM de $80 \mathrm{mmHg}$, seguido de RP60'.

Grupo Pringle: manobra de Pringle (MP) por 15 minutos, com PAM mantida em 40 mmHg com reposição de $\mathrm{RL}$ aquecido $\left(39-40^{\circ} \mathrm{C}\right)$, seguida de RP60’. 
Grupo Choque-Pringle: em estado de CHC com PAM mantida em $40 \mathrm{mmHg}$ por 20', seguido imediatamente de laparotomia e MP por 15', com reposição volêmica com RL aquecido $\left(39-40^{\circ} \mathrm{C}\right)$ e sangue total para PAM controlada em 40 mmHg durante a manobra, seguido de RP60'.

A volemia estimada do rato corresponde a $5,4 \mathrm{ml} / 100 \mathrm{~g}$ de peso. A quantidade de sangue de cada retirada inicialmente correspondeu a 5\% da volemia total estimada do animal até atingir ums PAM de $60 \mathrm{mmHg}$, ponto a partir do qual se passou para 2,5\% da volemia total para cada retirada até obter PAM de $40 \mathrm{mmHg}^{16}$. O volume de cristalóide em cada reposição correspondia a 5\% da volemia estimada do animal. Após três seqüências, utilizou-se o mesmo volume em sangue total. A laparotomia foi realizada por uma incisão mediana, sendo localizado o ligamento hepatoduodenal, que contém a Tríade Portal (veia porta, artéria hepática e ducto biliar). No ligamento realizou-se a $\mathrm{MP}$, que corresponde ao pinçamento do ligamento, com microclip vascular hemostático atraumático delicado, por um período constante de 15', exceto nos animais dos Grupos Controle e Choque. Após o período da MP, foi realizada a RP60, com reposições volêmicas de RL aquecido e sangue total para manutenção da PAM maior que $60 \mathrm{mmHg}$. Em cada fase do experimento de todos os grupos foram monitorizadas as seguintes variáveis através do Polígrafo Mingograf 807 (Siemens-Elema, Alemanha): PAM, freqüência cardíaca (FC), eletrocardiograma (ECG) e temperatura retal (TR). As amostras de sangue para gasometria, dosagens do LS e dos eletrólitos foram obtidas pela artéria carótida direita. As fases do experimento nas quais foram avaliados e comparados as variáveis foram: início do estudo (SVI), início da reperfusão (RP0), trinta minutos de reperfusão (RP30) e RP60. Todos os animais, após RP60 foram sacrificados por exsangüinação pela artéria carótida direita. A aorta abdominal foi clampleada e a veia cava inferior seccionada. Injetou-se $40 \mathrm{ml}$ de soro fisiológico cuidadosamente pela artéria femoral direita, realizando-se a limpeza do leito vascular dos órgãos abdominais. O rim direito foi retirado e fixado em formalina à $10 \%$, sendo a metade da porção do pólo superior foi enviada para embebição em parafina, da qual foram realizados cortes de cinco micra de espessura e corados com da técnica de Hematoxilina e Eosina (HE). A contagem de polimorfonucleares (PMN) no rim foi realizada no interstício do córtex. Foram examinados dez campos utilizando objetiva 100 e ocular de 10, determinando o número médio/campo. Os campos examinados obedeceram a seguinte regra: examinava-se um campo e saltavam-se dois campos correspondentes iguais para que se examinasse o próximo, seqüencialmente. O LS foi determinado pela utilização de teste BM-Lactateâ (Roche), que consiste em tiras testes para determinação quantitativa utilizando o fotômetro de reflexão pela reação colorimétrica do mediador lactatooxidase. As determinações sangüíneas do $\mathrm{pH}, \mathrm{RB}, \mathrm{pCO}_{2}$, $\mathrm{pO}_{2}, \mathrm{HCO}_{3}^{-}$, potássio, sódio, hematócrito $(\mathrm{Ht})$ e hemoglobina (Hb) foram realizados no aparelho de gasometria da Instrumental Laborato. Os resultados foram comparados e a significância estatística foi avaliada por meio da Análise de Variância (Anova de um critério - Teste F). Para avaliar os resultados em função dos fatores grupos e tempo foi utilizada a Análise de Variância com medidas repetidas. Com base na significância de cada efeito foi avaliada a diferença entre os grupos ou entre os tempos. Quando a interação foi significativa, realizou-se o teste de comparação (Teste de Tukey) entre as médias de um fator para cada nível do outro fator. Foi utilizado o nível de significância de 5\% (p $£$ 0,05) e os valores foram representados sob a forma de média \pm desvio padrão da média.

\section{Resultados}

Os pesos dos animais entre os grupos não apresentaram diferenças significativas (Anova: p=0,258). Os valores da média e desvio-padrão das variáveis no início do experimento não apresentaram diferenças estatísticas. Antes da realização da MP as alças intestinais não apresentavam estase venosa (Figura 1A). Porém, nos primeiros minutos após a MP observou-se intensa congestão venosa no leito esplâncnico (Figura 1B), caracterizada pela dilatação venosa e cianose nos órgãos

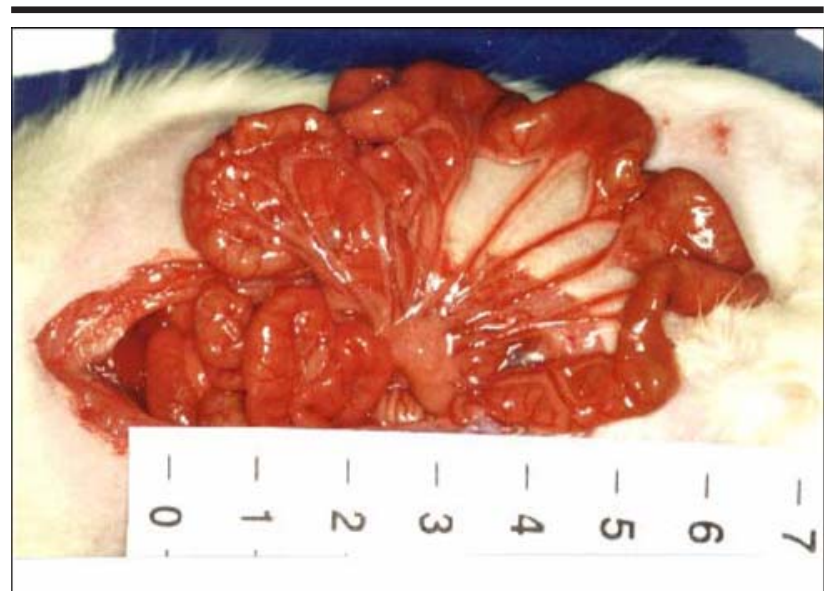

FIGURA 1A - Aspecto das alças intestinais antes da MP.

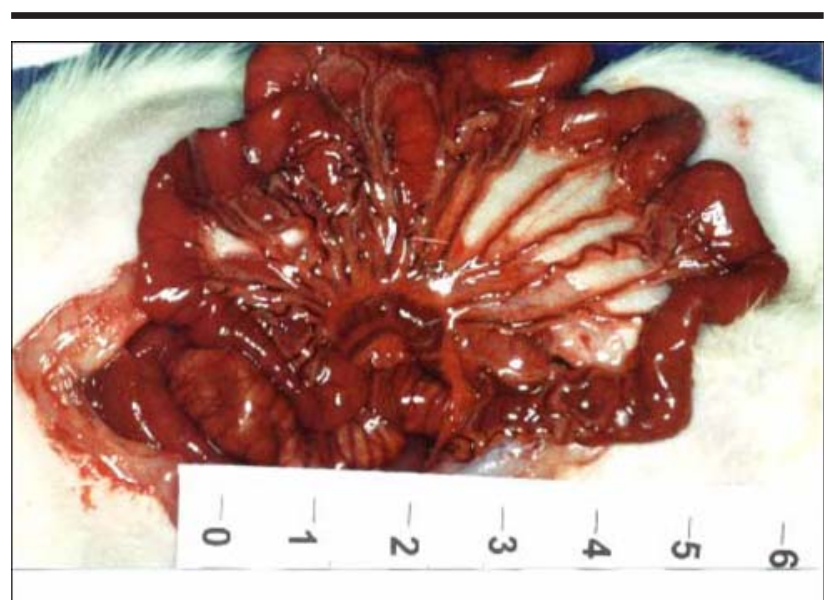

FIGURA 1B - Aspecto das alças intestinais nos primeiros minutos após a MP Observa-se intensa congestão venosa.

como baço e intestino delgado.

As variáveis relacionadas ao estado de choque hemorrágico incluíram as medidas do pH, RB e LS. A tabela 1 mostra os valores médios do $\mathrm{pH}$ em cada um dos grupos estudados. A análise estatística foi feita nas seguintes fases: SVI, RP0, RP30 e RP60. No decorrer de todo o experimento 
não foram verificadas diferenças estatísticas entre os grupos. A tabela 2 demonstra os valores médios da RB em cada um dos grupos estudados. A análise estatística foi feita nas seguintes fases: SVI, RP0, RP30 e RP60. A análise mostrou que no SVI os valores da RB não apresentaram valores estatisticamente diferentes entre si $(p=0,161)$. Na $\mathrm{RP} 0$, apenas o grupo Controle, com valor dentro dos limites de normalidade $(0,02 \mathrm{mmol} / \mathrm{l})$, diferiu dos demais grupos $(\mathrm{p}<0,0001)$, os quais apresentavam valores abaixo desse limite (Choque $=-8,21 \mathrm{mmol} / \mathrm{l}$, Pringle $=-7,52 \mathrm{mmol} / \mathrm{l}$, ChoquePringle $=-6,04 \mathrm{mmol} / \mathrm{l})$. No RP30 os valores dessa variável foram significativamente diferentes entre todos os grupos $(p<0,0001)$. Nessa fase, apenas os animais do grupo Choque apresentaram um retorno da $\mathrm{RB}$ próximo à normalidade (BE $=-3,55 \mathrm{mmol} / \mathrm{l})$. No RP60, apenas o grupo Pringle apresentou valor estatisticamente diferente dos demais $(\mathrm{p}<0,001)$. A tabela 3 mostra os valores médios do LS em cada um dos grupos estudados. A análise estatística foi feita nas seguintes fases: SVI, RP0, RP30 e RP60. O LS no SVI não apresentou diferença estatística entre os grupos $(p=0,678)$. $\mathrm{Na}$ RP0, apenas o grupo Controle, com $0.92 \mathrm{mmol} / \mathrm{l}$, apresentou diferença estatística dos demais $(\mathrm{p}<0,0001)$, os quais apresentaram valores bem acima dos limites de normalidade (Choque $=4,16 \mathrm{mmol} / \mathrm{l}$, Pringle $=3,58 \mathrm{mmol} / \mathrm{l}$, Choque-Pringle $=4,15 \mathrm{mmol} / \mathrm{l})$. Na RP30 os grupos Pringle $\mathrm{e}$ Choque-Pringle diferiram significativamente entre si $(\mathrm{p}<0,0001)$. O grupo Pringle foi o que apresentou menor redução do valor desta variável no sentido da normalidade, permanecendo sempre elevado ao longo de todo o período de reperfusão. O grupo Controle na RP60 manteve-se dentro da normalidade diferenciando estatísticamente dos demais grupos $(\mathrm{p}=0,009)$. O número de neutrófilos por campo, presente no córtex renal, em cada grupo, após RP60', está representado na tabela 4. A análise de comparação múltipla mostrou que, após o período RP60, ocorreu diferença estatisticamente significativa quanto aos valores relativos às contagens de neutrófilos por campo nos diferentes grupos estudados $(\mathrm{p}<0,001)$. Na análise de comparação dois a dois, verifica-se que apenas o grupo Choque apresentou diferença significativa quando comparado aos demais, os quais, não diferiram entre si.

TABELA 1 - Valores médios do pH em cada fase do experimento conforme o grupo estudado

\begin{tabular}{llllll}
\hline Grupo & & SVI & RP0 & RP30 & RP60 \\
\hline \multirow{4}{*}{ Controle } & Média & 7,329 & 7,329 & 7,280 & 7,302 \\
& DP & 0,062 & 0,062 & 0,090 & 0,075 \\
& N & 8 & 8 & 8 & 8 \\
\hline \multirow{4}{*}{ Choque } & Média & 7,257 & 7,272 & 7,320 & 7,299 \\
& DP & 0,059 & 0,043 & 0,049 & 0,077 \\
& N & 8 & 8 & 8 & 8 \\
\hline \multirow{3}{*}{ Pringle } & Média & 7,311 & 7,308 & 7,256 & 7,199 \\
& DP & 0,049 & 0,084 & 0,098 & 0,126 \\
& N & 8 & 8 & 8 & 7 \\
\hline \multirow{2}{*}{ Choque- } & DP & 0,033 & 0,031 & 0,022 & 0,082 \\
Pringle & N & 8 & 7 & 7 & 6 \\
P* & & 0,059 & 0,110 & 0,187 & 0,104 \\
\hline
\end{tabular}

*Análise de Variância (Anova).
TABELA 2 - Valores médios da RB em cada fase do experimento conforme o grupo estudado.

\begin{tabular}{|c|c|c|c|c|c|}
\hline Grupo & & SVI & RP0 & RP30 & RP60 \\
\hline \multirow[t]{3}{*}{ Controle } & $\begin{array}{l}\text { Média } \\
(\mathrm{mmol} / \mathrm{l})\end{array}$ & 0,02 & 0,02 & $-1,52$ & $-2,63$ \\
\hline & DP & 2,18 & 2,18 & 1,93 & 2,38 \\
\hline & $\mathbf{N}$ & 8 & 8 & 8 & 8 \\
\hline \multirow[t]{3}{*}{ Choque } & $\begin{array}{l}\text { Média } \\
(\mathrm{mmol} / \mathrm{l})\end{array}$ & $-2,23$ & $-8,21$ & $-3,55$ & $-4,66$ \\
\hline & DP & 1,90 & 2,13 & 1,85 & 2,81 \\
\hline & $\mathbf{N}$ & 8 & 8 & 8 & 8 \\
\hline \multirow[t]{3}{*}{ Pringle } & $\begin{array}{l}\text { Média } \\
(\mathrm{mmol} / \mathrm{l})\end{array}$ & $-1,46$ & $-7,52$ & $-10,07$ & $-10,94$ \\
\hline & DP & 2,37 & 3,01 & 1,70 & 3,50 \\
\hline & $\mathbf{N}$ & 8 & 8 & 8 & 7 \\
\hline \multirow{3}{*}{$\begin{array}{l}\text { Choque- } \\
\text { Pringle }\end{array}$} & $\begin{array}{l}\text { Média } \\
(\mathrm{mmol} / \mathrm{l})\end{array}$ & $-1,00$ & $-6,04$ & $-5,98$ & $-6,56$ \\
\hline & DP & 1,15 & 1,79 & 1,93 & 3,19 \\
\hline & $\mathbf{N}$ & 8 & 7 & 7 & 6 \\
\hline$P^{*}$ & & 0.161 & 0,000 & 0,000 & 0,000 \\
\hline
\end{tabular}

* Análise de Variância (ANOVA).

TABELA 3 - Valores médios do LS em cada fase do experimento conforme o grupo estudado.

\begin{tabular}{|c|c|c|c|c|c|}
\hline Grupo & & SVI & RP0 & RP30 & RP60 \\
\hline \multirow[t]{3}{*}{ Controle } & $\begin{array}{l}\text { Média } \\
(\mathrm{mmol} / \mathrm{)})\end{array}$ & 0,92 & 0,92 & 1,05 & 0,95 \\
\hline & DP & 0,12 & 0,12 & 0,23 & 0,15 \\
\hline & $\mathbf{N}$ & 8 & 8 & 8 & 8 \\
\hline \multirow[t]{3}{*}{ Choque } & $\begin{array}{l}\text { Média } \\
(\mathrm{mmol} / \mathrm{l})\end{array}$ & 0,95 & 4,16 & 1,68 & 3,58 \\
\hline & DP & 0,19 & 1,30 & 0,77 & 2,76 \\
\hline & $\mathbf{N}$ & 8 & 8 & 8 & 8 \\
\hline \multirow[t]{3}{*}{ Pringle } & $\begin{array}{l}\text { Média } \\
(\mathrm{mmol} / \mathrm{l})\end{array}$ & 0,91 & 3,58 & 3,13 & 6,55 \\
\hline & DP & 0,20 & 0,67 & 0,32 & 4,98 \\
\hline & $\mathbf{N}$ & 8 & 8 & 8 & 7 \\
\hline Choque- & $\begin{array}{l}\text { Média } \\
\text { (mmol/l) }\end{array}$ & 0,85 & 4,15 & 2,90 & 3,03 \\
\hline \multirow[t]{2}{*}{ Pringle } & DP & 0,14 & 0,57 & 0,69 & 1,01 \\
\hline & $\mathbf{N}$ & 8 & 7 & 7 & \\
\hline$P^{*}$ & & 0.678 & 0.000 & 0.000 & 0.009 \\
\hline
\end{tabular}

*Análise de Variância (Anova)

Durante o experimento morreram três (9,3\%) animais. A tabela 5 mostra o número de óbitos, desses animais, em cada um dos grupos estudados.

A análise estatística não mostrou diferença significativa no número de mortes por grupo e, no caso das mortes $(n=2)$ no grupo Choque-Pringle, uma delas ocorreu durante a fase de isquemia hepática, mostrando, dessa maneira, que das três mortes ocorridas na presente pesquisa, duas ocorreram durante a MP. 
TABELA 4 - Número de neutrófilos, por campo, no rim, conforme o grupo estudado

\begin{tabular}{lllll}
\hline Grupo & Controle & Choque & Pringle Choque-Pringle \\
\hline PMN/Campo & & & & \\
Média & 0,24 & $\mathbf{1 , 0 6}$ & 0,18 & 0,24 \\
DP & 0,28 & $\mathbf{0 , 6 1}$ & 0,16 & 0,19 \\
N & 8 & $\mathbf{8}$ & 7 & 6 \\
\hline
\end{tabular}

Anova: $\mathbf{p}<0,001$

TABELA 5 - Número de óbitos conforme o grupo estudado

\begin{tabular}{|c|c|c|c|c|c|c|c|c|c|}
\hline \multirow{2}{*}{$\begin{array}{l}\text { Grupo } \\
\text { Óbito }\end{array}$} & \multicolumn{2}{|c|}{ Controle } & \multicolumn{2}{|c|}{ Choque } & \multicolumn{2}{|c|}{ Pringle } & \multicolumn{2}{|c|}{ Choque-Pringle } & \multirow[b]{2}{*}{ Total } \\
\hline & $\mathbf{n}^{0}$ & $\%$ & & $\%$ & & $\%$ & n. $^{\mathbf{o}}$ & $\%$ & \\
\hline Sim & 0,0 & 0,0 & 0,0 & 0,0 & 01 & 12,5 & 02 & 25,0 & 03 \\
\hline Não & 08 & 100,0 & 08 & 100,0 & 07 & 87,5 & 06 & 75,0 & 29 \\
\hline Total & \multicolumn{2}{|c|}{08} & \multicolumn{2}{|c|}{08} & \multicolumn{2}{|c|}{08} & \multicolumn{2}{|c|}{08} & 32 \\
\hline
\end{tabular}

\section{Discussão}

A forma de estabelecimento do CHC foi realizada com retiradas de $5 \%$ da volemia estimada até PAM de $60 \mathrm{mmHg}$ com intervalo de 2 minutos entre cada sangria. A partir desse ponto, as retiradas foram de $2,5 \%$ da volemia estimada até PAM de $40 \mathrm{mmHg}$. Essa padronização, conforme a literatura tem como objetivo simular uma lesão hemorrágica, tendo em vista que conforme a queda da PAM diminui o fluxo de sangramento pela lesão ${ }^{16}$. Na literatura há vários estudos sobre as diversas soluções para reanimação volêmica. Entretanto, nos serviços de urgência e emergência a solução habitualmente utilizada na prática clínica é o RL, motivo pelo qual foi padronizada no protocolo. Estudo anterior demonstra a curva de sobrevida desse animal quando submetido à MP. Quando se realizava um período de isquemia hepática de 15’ havia 95\% de sobreviventes e 91\% quando se submetia a 30 minutos $^{10,23}$. Esses resultados demonstram que o rato é um animal adequado para este modelo experimental, e, por isso, o mais utilizado para estudo da isquemia e reperfusão hepática. E diante da necessidade de uma taxa de mortalidade baixa foi padronizado o tempo de MP em $15^{16}$. O fígado humano habitualmente pode tolerar, sob normotermia, um período de isquemia acima de 20 minutos $^{7}$. Relatam, em estudo clínico, vítimas de traumatismo hepático grave (graus IV-VI) que foram submetidos à MP com tempo médio de 32 minutos ( 10 a 75 minutos) sem que ocorresse lesão do órgão por isquemia. Há outros relatos na literatura que variaram de 25 minutos $^{24}$ a 180 minutos $^{25}$. Logo após a MP, a presente pesquisa verificou hipotensão arterial e congestão venosa do leito esplâncnico, alterações estas já descritas na literatura ${ }^{9,10}$. O período de reperfusão preconizado, conforme a literatura, é tópico de discussão, assim como ocorre na determinação do tempo ideal da MP. ${ }^{26}$ Utilizaram um tempo de reperfusão de 0 minutos e em outro estudo, foi de 21 dias $^{27}$. O tempo de reperfusão utilizado de 60 minutos neste trabalho foi determinado diante da necessidade de se estabelecer uma correlação com as alterações que poderiam ser encontradas após a MP associadas ao estado de normovolemia e hipovolemia. A escolha adequada da droga e a via de administração para procedimento anestésico são fatores importantes que podem influenciar nas interpretações, por exemplo, de variáveis hemodinâmicas durante o ato cirúrgico no decorrer de um modelo experimental. Conforme a literatura ${ }^{9,22}$ na escolha dos agentes anestésicos adequados para o protocolo do presente experimento foi selecionada a associação de Cetamina/Xilazina/atropina, por inoculação intraperitoneal. Estudos relatam que os parâmetros clínicos como pressão arterial, PVC e FC são freqüentemente utilizados para avaliar o estado de choque. Porém, estes índices podem sofrer alterações devido à resposta compensatória e às medicações administradas ${ }^{28,29}$. A normalização da PVC após um período de choque pode levar a um estado de hipervolemia ${ }^{30}$. Quando os tecidos estão em condições de má perfusão, as células, para manterem um nível adequado de energia para o seu metabolismo, entram no ciclo anaeróbico, onde ocorre a utilização do piruvato com conseqüente fornecimento de duas moléculas de ATP e lactato ${ }^{1,17}$. Em estudo clínico ou experimental do CH ocorre a acidose metabólica decorrente da isquemia tecidual. Essa situação de metabolismo anaeróbico é responsável pela elevação do nível de lactato ${ }^{9,13,14,15,28}$. Na acidose metabólica ocorre também o consumo de RB ("base excess”) como meio de compensação da alteração do pH sangüíneo. As determinações do LS e RB podem ser utilizadas como parâmetros de hipoperfusão tecidual e também para avaliar a reanimação ${ }^{9,17}$. A mensuração do LS na prática médica não é um exame rápido e de rotina nos laboratórios da maioria das instituições, no atendimento inicial ao politraumatizado, apesar de ser considerado na literatura como um ótimo indicador de estado de choque e de reanimação $0^{9,13-15,28}$. No presente trabalho utilizou-se tiras testes para determinação quantitativa do LS que é um método de fácil aplicação. Outra forma de mensuração é através de equipamento de gasometria que, além dos parâmetros dos gases sangüíneos completos, determinam medidas de eletrólitos, glicemia, hemoglobina, hematócrito e o lactato. A determinação da RB é outro método utilizado como índice para avaliar o estado de choque. O volume de cristalóides e/ou sangue utilizados na reanimação é diretamente proporcional ao déficit de base ${ }^{17,28,31}$. O grupo Pringle demonstrou os maiores valores do lactato sérico (RP 60 = 6,55 mmol/l) e da RB (RP 
$60=-10,94 \mathrm{mmol} / \mathrm{l})$ no final do experimento, caracterizando o estado de má perfusão tecidual ocorrido no animal. A I/R hepática tem sido modelo em estudo de diversos trabalhos na literatura, como alavanca inicial no desenvolvimento da SDMO, porém sem associação do estado de hipovolemia9 . MARTINS $^{32}$ utilizou a MP como modelo experimental de isquemia e reperfusão sem hipovolemia, procedimento que mesmo assim conduz a uma isquemia renal por diminuição do débito cardíaco em conseqüência da queda da pré-carga. Nesse estudo, a maior ocorrência de seqüestro de neutrófilos, através da dosagem da mieloperoxidase, foi verificado no grupo submetido a um período de reperfusão de 120 minutos. As alterações funcionais e morfológicas do rim na SDMO ocorrem devido à hipoperfusão tecidual, e a redistribuição do fluxo sangüíneo afeta primeiramente o córtex renal ${ }^{1}$. Foi demonstrado, em estudo experimental, que a isquemia renal seguida de reperfusão é responsável pela lesão tecidual independentemente das alterações imunológicas, e condutas que previnam ou amenizem essas situações são prioridade como proteção renal ${ }^{33}$. Após a isquemia e reperfusão renal ocorre necrose tubular aguda nas primeiras horas, com ausência de infiltrado celular, porém com ativação de células inflamatórias (polimorfonucleares, monócitos/macrófagos). Posteriormente, há regeneração das lesões com conseqüente acúmulo de monócitos/macrófagos e linfócitos $\mathrm{T}$, em contraste com o baixo número de neutrófilos encontrados. Os métodos de identificação específica de neutrófilos (exs: mieloperoxidase) fazem reação cruzada com monócito/ macrófago, fato que esclarece o menor número presente de neutrófilos pós isquemia e reperfusão renal ${ }^{34}$. A determinação do nível de mieloperoxidase para presença de PMN no tecido é limitado por outros motivos: a mensuração da enzima não distingue PMN aderidos e circulantes; na concentração total da enzima há participação de peroxidases e outras mieloperoxidases tal como hemoglobina “pseudoperoxidase" e catalase ${ }^{35}$. Devido aos fatores que podem influenciar uma avaliação bioquímica adequada do seqüestro de neutrófilos no interstício dos tecidos, a contagem dos mesmos é um indíce fiel sem interferência dos fatores acima citados. Neste estudo, ocorreu acúmulo máximo de PMN no córtex renal no grupo Choque (1,06 \pm $0,61 \mathrm{PMNs} / \mathrm{campo}$ ). Tal resultado foi inesperado, visto que o CH seguido pela MP, duas condições críticas que foram estabelecidas no grupo Choque-Pringle, não promoveram maior acúmulo de neutrófilos em comparação aos grupos Choque e Pringle. Este fato por ser explicado pelas alterações hemodinâmicas presentes durante a MP caracterizadas pela queda abrupta do débito cardíaco e volume circulante efetivo, fatores que promovem uma diminuição do fluxo sangüíneo renal, conseqüentemente um menor número de neutrófilos. Como se sabe, a isquemia tecidual provoca uma lesão endotelial desencadeando o processo de seqüestração de neutrófilos. Dessa forma, após a MP, os neutrófilos ativados no território esplâncnico percorrem o leito vascular hepático, local onde realizam aderência ao endotélio decorrente do processo de I/R hepática, o que proporciona uma diminuição dessas células ativadas carreadas pela corrente sangüínea. Essa condição contribui para um menor acúmulo de neutrófilos no rim mediante a MP. Outro fator relevante que é responsável pelo efeito de seqüestração dos neutrófilos após isquemia tecidual é o período de reperfusão. MARTINS ${ }^{32}$ demonstrou que o afluxo de neutrófilos é tempo-dependente para o rim. Após um período de 120 minutos de reperfusão, inicia-se um afluxo maior dessas células para o interstício. No presente trabalho, ocorreram três óbitos (9,3\%): dois animais no grupo Choque-Pringle e um no grupo Pringle. Em todos eles foram realizadas as necrópsias, em que foram verificadas as seguintes alterações macroscópicas: congestão venosa esplâncnica importante, petéquias nas alças intestinais e exsudato sanguinolento na cavidade abdominal. $\mathrm{Na}$ literatura, estudos demonstraram que a MP quando realizada acima de um período maior que 30 minutos em ratos é potencialmente letal ${ }^{10,36}$. No trauma, o atendimento inicial e a reanimação, seguidos pelo reparo das lesões, são os primeiros degraus do tratamento efetivo do paciente. $\mathrm{O}$ cuidado ao paciente traumatizado crítico no período pósoperatório imediato e tardio concentra-se no controle das repercussões e complicações das lesões reparadas com ou sem condições associadas (patologias mórbidas e/ou situação crítica como o $\mathrm{CH}$ ). O trauma hepático grave é tema de constantes estudos devido às complicações inerentes à lesão e ao estado de $\mathrm{CH}$ que apresentam um índice elevado de morbidade e mortalidade. O presente trabalho estabelece um modelo experimental de duas situações críticas no trauma: I/R hepática e o $\mathrm{CH}$. Este modelo, baseando-se na revisão de literatura, tem como finalidade avaliar os princípios das alterações sistêmicas que desencadeiam a SDMO tendo, como ponto de referência, a lesão renal. Conforme a literatura, os neutrófilos têm participação fundamental na lesão dos órgãos no desenvolvimento da SDMO, mediante sua ativação seguida da seqüestração no interstício dos tecidos. Portanto, a partir desses princípios e diante dos resultados deste trabalho experimental, torna-se primordial que novos estudos sejam realizados para melhor compreensão dos mecanismos de lesão tecidual provocada tanto no estado de má perfusão ou no período de reperfusão, assim como estabelecer protocolos que venham prevenir ou amenizar as complicações tardias dessas situações críticas no paciente traumatizado.

\section{Conclusão}

A avaliação dos resultados do presente modelo experimental demonstrou que no grupo Choque ocorreu maior seqüestro de polimorfonucleares comparativamente aos demais grupos. E nos grupos Pringle e Choque-Pringle, comparativamente, não foram significativas a ocorrência de seqüestro de polimorfonucleares.

\section{Referências}

1. Andersson RW, Vaslef SN - Choque: causas e controle do colapso circulatório. In: SABBISTON, D. C. Jr. Tratado de Cirurgia: as bases biológicas da prática cirúrgica moderna. 15.ed., Rio de Janeiro, Guanabara-Koogan, 1999. p.63-85, vol.1.

2. Datasus - http://www.datasus.gov.br

3. Bitterman H, Brod V, Weisz G, Kushnir D, Bitterman N Effects of oxigen on regional hemodynamics in hemorrhagic 
shock. Am J Physiol. 1996; 271(1Pt2):H203-11.

4. Silva MR, Figueiredo LFP - Fisiopatologia do choque hipovolêmico. In: Younes RN, Birolini D (ed). - Bases fisiopatológicas da cirurgia. São Paulo, Lemar, 1999. p.9-19.

5. Poggetti RS, Souza Jr AL, Fontes B. - Isquemia e reperfusão. In: Younes RN, Birolini D (ed). - Bases fisiopatológicas da cirurgia. São Paulo, Lemar, 1999. p.135-47.

6. Feliciano DV, Mattox KL, Jordan GL Jr, Burch JM, Bitondo CG, Cruse PA - Management of 1000 consecutive cases of hepatics trauma (1979-1984). Ann Surg. 1986;204:438-45.

7. Pachter HL, Spencer FC, Hoffstetter SR, Liang HG, Coppa GF - Significant trends in the treatment of hepatic trauma. Experience with 411 injuries. Ann Surg. 1992;215:492-502.

8. Pringle $\mathrm{JH}$ - Notes on the arrest of hepatic hemorrhage due to trauma. Ann Surg. 1908;48: 541-9.

9. Hirano ES, Morandin RC, Mantovani M - Efeitos da manobra de Pringle nos níveis pressóricos em condições de normalidade e estado de choque hemorrágico controlado no rato. Acta Cir Bras. 1999;14(supl. 2):69.

10. Gonce ME, Brackett DJ, Squires RA, Gibson DD, Balla AK, Lerner MR, McCay PB, Pennington LR Development of circulatory and metabolic shock following transient portal triad occlusion. J Surgery Res. 1995;59:534-43.

11. Huckabee WE - Relationships of pyruvate and lactate during anaerobic metabolism: II. Exercise and formation of $\mathrm{O}_{2}$ debt. J Clin Invest. 1958;37:255-263.

12. Cady L, Weil MH, Abdelmonem AA - Quantitation of severity of critical illness with special reference to blood lactate. Crit Care Med. 1973;1:75-80.

13. Broder $\mathrm{G}$ \& Weil MH - Excess lactate: An index of reversibility of shock in human patients. Science. 1964;143:1457.

14. Blair E, Cowley RA, Tait M K - Refractory septic shock in man: role of lactate and pyruvate metabolism and acidbase balance in prognosis. Am Surg. 1965;31: 537.

15. Vitek V, Cowley RA - Blood lactate in the prognosis of various forms of shock. Ann Surg. 1971;173: 308-13.

16. Hirano ES, Mantovani M, Morandin RC, Fontelles MJP. Modelo experimental de choque hemorrágico. Acta Cir Bras. 2003;18:465-70.

17. Davis JW, Shackford SR, Mackersie RC, Hoyt DB - Base deficit as a guide to volume resuscitation. J Trauma. 1988;28:1464-67.

18. Burke TJ, Burnier M, Langberg H, Shanley P, Schrier RW - Renal response to shock. Ann Emerg Med. 1986;15: 1397-400.

19. Parks DA, Granger DN - Ischemia-reperfusion injury: a radical view. Hepatology. 1988;8:680-2, 1988

20. Botha AJ, Moore FA, Moore EE, Kim FJ, Banerjee A, Peterson VM - Postinjury neutrophil priming and activation: an early vulnerable window. Surgery. 1995;118:358-64.

21. Vedder NB, Fouty BW, Winn RK, Harlan JM, Rice CL Role of neutrophils in generalized reperfusion injury associated with resuscitation from shock. Surgery. 1989;106: 509-16.
22. Bacelar Jr JC, Repka JC, Eidt J, Fonseca VRCD, Salles Jr G, Souza-Lima MB - Avaliação de hidrato de cloral, tiopental sódico e associação cetamina/xilazina na anestesia em ratos. Acta Cir Bras. 1999;14:149.

23. Kogure K, Suzuki M - Effects of hepatic inflow occlusion on changes in plasma potassium, histamine, and norepinephrine in rats. Circ Shock. 1992;36:290-8.

24. Komatsu H, Koo A, Ghadishah E, Zeng H, Kuhlenkamp JF, Inoque M, Guth PH, Kaplowitz N - Neutrophil accumulation in ischemic reperfused rat liver: evidence for a role for superoxide free radicals. Am J Physiol. 1992;262:669-76.

25. Rodrigues AA, LaMorte WW, Hanrahan LM, Hopkins SR, O’Keane JC, Cachecho R, Hirsch EF - Liver viability after ischemia-reperfusion. Arch Surg. 126:767-72, 1991.

26. Colletti LM, Remick DG, Burtck GD, Kunkel SL, Streater RM, Campbell Jr DA - Role of tumor necrosis factor-a in the pathophysiologic alterations after hepatic ischemia/ reperfusion injury in the rat. J Clin Invest. 1990;85:1936-43.

27. Nauta RJ, Tsimoyannis E, Uribe M, Walsh DB, Miller D, Butterfield A -Oxigen-derived free radicals in hepatic ischemia and reperfusion injury in the rat. Surg Gynecol Obstet. 1990;171:120-5.

28. Davis JW - The relationship of base deficit to lactate in porcine hemorrhagic shock and resuscitation. J Trauma. 1994; 36:168-72.

29. Shah NS, Kelly E, Billiar TR, Marshall HM, Harbrecht BG, Udekwu AO, Peitzman AB - Utility of clinical parametes of tissue oxygenation in a quantitative model of irreversible hemorrhagic shock. Shock. 1998;10:3436.

30. McNamara JJ, Suehiro G T, Suehiro A, Jewett B Resuscitation from hemorrhagic shock. J Trauma. 1983;23:552-8.

31. Davis JW ,Shackford SR, Holbrook TL - Base deficit as a sensitive indicator of compensated shock and tissue oxygen utilization. Surg Gynecol Obstet. 1991;173: 473-6.

32. Martins $\mathrm{F}^{\circ}$, O. - Efeitos da isquemia e reperfusão hepática total no seqüestro de neutrófilos no pulmão, rim e íleo terminal e na produção de citocinas. Estudo experimental em ratos. São Paulo, 1997. (Tese-Doutorado-Faculdade de Medicina da Universidade de São Paulo).

33. Dragun D, Hoff U, Park JK, Qun Y, Schneider W, Luft FC, Haller H. - Ischemia-reperfusion injury in renal transplantation is independent of the immunologic background. Kidney Int. 2000; 58:2166-77, 2000.

34. Ysebaert DK, De Greef KE, Vercauteren SR, Ghielli M, Verpooten GA, Eyskens EJ, De Broe M.E. - Identification and kinetics of leukocytes after sever ischaemia/ reperfusion renal injury. Nephrol Dial Transplant. 2000;15:1562-74.

35. Anderson BO, Brown JM, Shanley PF, Bensard DD, Harken AH - Marginating neutrophils are reversibly adherent to normal lung endothelium. Surgery. 1991;109:51-61.

36. Meija R H - Hypovolemic shock in the rat. Acta Physiol Lat Am 1964;14:7. 


\section{Correspondência:}

Elcio Shiyoiti Hirano

Av. Governador Pedro de Toledo, 638 apto 91-3

13070-752 - Bonfim - Campinas-SP

elciohirano@ig.com.br
Conflito de interesse: nenhum

Fonte de financiamento: nenhuma

Recebimento: 02/03/2005

Revisão: 12/04/2005

Aprovação: 17/05/2005

\section{Como citar este artigo:}

Hirano ES, Mantovani M, Morandin RC. Isquemia e reperfusão hepática total em condições de normalidade e sob estado de choque hemorrágico controlado: efeitos no sequestro de neutrófilos no rim do rato. Acta Cir Bras. [periódico na internet] 2005 Jul-Ago;20(4). Disponível em URL: $\underline{\text { http://www.scielo.br/acb }}$

* Figuras coloridas disponíveis em http://www.scielo.br/acb

\section{VISITE O NOSSO SITE}

\section{SOCIEDADE BRASILEIRA PARA O DESENVOLVIMENTO DA PESQUISA EM CIRURGIA SOBRADPEC}

http://www.sobradpec.org.br 